\title{
Predictors of clinical outcomes after periodontal treatment of aggressive periodontitis: 12-month randomized trial
}

\section{Alex Nogueira HAAS(a) \\ Carina Maciel \\ SILVA-BOGHOSSIAN ${ }^{(b)}$ \\ Ana Paula COLOMBO(b) \\ Jasim ALBANDAR ${ }^{(c)}$ \\ Rui Vicente OPPERMANN(a) Cassiano Kuchenbecker RÖSING(a) Cristiano SUSIN ${ }^{(d)}$}

\footnotetext{
(a) Universidade Federal do Rio Grande do Sul, Faculty of Dentistry, Periodontology, Porto Alegre, Brazil.

(b) Universidade Federal do Rio de Janeiro - UFRJ, Institute of Microbiology, Rio de Janeiro, RJ, Brazil.

(c)Temple University, School of Dentistry, Department of Periodontology, Philadelphia, PA, USA.

(d) Georgia Regents University, Departments of Periodontics and Oral Biology, Augusta, GA, USA.
}

Declaration of Interests: The authors certify that they have no commercial or associative interest that represents a conflict of interest in connection with the manuscript.

\section{Corresponding Author:}

Alex Nogueira Haas

e-mail: alexnhaas@gmail.com

DOI: 10.1590/1807-3107BOR-2016.vol30.0041

Submitted: Jul 29, 2015

Accepted for publication: Oct 27, 2015

Last revision: Dec 17, 2015

\begin{abstract}
Little is known about the factors that may be used in clinical practice to predict the therapeutic response of aggressive periodontitis patients. The aim of this study was to determine predictors of clinical outcomes after non-surgical treatment of aggressive periodontitis. A total of 24 patients (aged 13-26 years) received oral hygiene instructions, as well as subgingival scaling and root planing. Twelve subjects received systemic azithromycin at random. Clinical variables were assessed at baseline, 3, 6, 9, and 12 months. Baseline microbiological assessment was performed by checkerboard DNA-DNA hybridization. Multivariable models used generalized estimating equations. There were significant improvements in the entire sample in regard to pocket depth, clinical attachment level and bleeding on probing. Significant predictors of a reduction in mean pocket depth were: use of azithromycin, non-molar teeth, generalized disease and baseline pocket depth. Absence of plaque predicted a $0.22 \mathrm{~mm}$ higher attachment gain, whereas a baseline pocket depth $\geq 7 \mathrm{~mm}$ predicted a $1.36 \mathrm{~mm}$ higher attachment loss. Azithromycin, plaque, and baseline pocket depth were significant predictors of bleeding on probing. The concomitant presence of all three red complex species predicted a $0.78 \mathrm{~mm}$ higher attachment loss. It may be concluded that dental plaque, tooth type, disease extent, baseline pocket depth, and use of azithromycin were significant predictors of the clinical response to treatment for aggressive periodontitis in young individuals. Moreover, the presence of multiple periodontal pathogens may predict challenges in achieving a favorable outcome for aggressive periodontitis.
\end{abstract}

Keywords: Aggressive Periodontitis; Prognosis; Anti-Bacterial Agents; Microbiology.

\section{Introduction}

Although the prevalence of aggressive periodontitis (AgP) is lower ${ }^{1,2}$ than that of chronic periodontitis, it still represents a challenge in periodontal practice. ${ }^{3}$ Studies have shown that periodontal treatment results in mixed clinical outcomes. ${ }^{4,5}$ Moreover, little is known about the factors that may contribute to a favorable outcome following periodontal treatment of AgP. Furthermore, the evidence available on treatment outcomes has been provided primarily by uncontrolled studies and short follow-ups.

Only two long-term interventional studies have evaluated prognostic factors among subjects with $\mathrm{AgP}^{6,7}$ One study involving $25 \mathrm{AgP}$ patients 
found that smoking, stress and counts of Porphyromonas gingivalis and Treponema denticola were significantly associated with disease progression. The other ${ }^{6}$ demonstrated that smoking and gingival bleeding were significantly associated with the progression of tissue loss. Tooth-related factors such as plaque, ${ }^{8,9}$ tooth type ${ }^{8}$ and baseline pocket depth ${ }^{10}$ have also been studied as predictors of clinical outcomes after periodontal therapy in general and in AgP. These studies have provided useful overall information; however, prospective randomized studies with a greater follow-up may provide clinicians with stronger evidence on the predictability of periodontal treatment for AgP.

Given this scenario, clinicians are frequently uncertain about how $\mathrm{AgP}$ patients will respond to periodontal treatment, and, just as importantly, about what factors can predict clinical outcomes. The aim of this study was to assess the significance of patient- and tooth-related predictors of 12-month outcomes after nonsurgical periodontal treatment of $\mathrm{AgP}$.

\section{Methodology}

\section{Study sample}

This study comprises a secondary analysis of a previously published randomized controlled trial. Detailed information about the study sample can be assessed in a previous publication of this randomized controlled trial. ${ }^{11}$

In brief, 24 AgP subjects, aged 13-26 years, with at least one incisor and one first molar with a periodontal probing depth (PPD) $\geq 4 \mathrm{~mm}$, CA loss $\geq 4$ $\mathrm{mm}$ and bleeding on probing (BOP) were followed for 12 months in the present study. Individuals were recruited from the Periodontal Department of the Universidade Federal do Rio Grande do Sul - UFRGS, Porto Alegre, Brazil.

\section{Interventions}

Periodontal treatment consisted of two sessions at one-week intervals of supragingival scaling and oral hygiene instructions, followed by subgingival scaling and root planing (SRP) under local anesthesia performed within a period of 14 days by an experienced periodontist. Twelve randomly selected subjects were administered azithromycin in $500 \mathrm{mg}$ tablets
(EMS-SIGMA, São Paulo, Brazil) once daily for 3 days, whereas the other subjects were given placebo tablets.

The subjects were stratified by smoking status (smokers or nonsmokers) and disease extent (localized or generalized) to allow similar distribution of these factors in the sample. An assistant not involved in the study was responsible for the randomization of the participants within each stratum. Randomization was conducted by a draw. Test (azithromycin) and control (placebo) medications were stored in identical opaque-colored bottles identified only by the respective code of each participant. Randomization codes were kept by the same assistant and were revealed only after the statistical analyses had been performed. The periodontists involved in the treatment and in the clinical examination were masked to the study participants allocation throughout the experimental period.

The subjects took the first tablet just before the first session of SRP. After treatment, they were seen every three weeks for the first three months, then once a month for the next 3 to 6 months, and, lastly, once every three months in the last 6 months of the study. Recall visits included oral prophylaxis and oral hygiene instructions on an individualized basis.

\section{Clinical examination}

Visible plaque (VP), PPD, gingival recession (GR) and $\mathrm{BOP}$ were assessed in all participants at baseline and at 3, 6, 9, and 12 months after treatment. One calibrated periodontist, blinded for the use of antibiotics, performed all the measurements on six sites per tooth, excluding third molars. PPD and GR were assessed using a manual periodontal probe (CP10SE, HuFriedy, Chicago, USA). The CA level was calculated as the sum of probing depth and gingival recession. BOP was recorded as present/absent after PPD measurements.

Repeated measurements were performed on 12 subjects to evaluate the examiner reproducibility, with at least one hour between each examination. The weighted kappa coefficients $(1 \mathrm{~mm})$ of PPD and CA loss were 0.92 and 0.85 , respectively.

\section{Sample size}

The sample size was calculated based on the aims of the previous publication of this clinical trial. ${ }^{11}$ A sample size of 24 subjects was estimated 
as needed to achieve an $80 \%$ power to detect a mean PPD difference of $1 \mathrm{~mm}$ (standard deviation 0.85) between the azithromycin and the placebo groups. A two-sided two-sample t-test with a significance level of $5 \%$ was used to calculate the sample size.

\section{Microbiology}

One pool of subgingival plaque was obtained for each participant at baseline. The supragingival plaque was removed, and subgingival samples were taken with sterile paper points from the deepest site of each tooth from all the teeth present. The samples were placed in Eppendorf tubes containing $1 \mathrm{ml}$ of TE buffer (10 mM Tris-HCl, 0.1 mM EDTA, $\mathrm{pH} 7.6)$ and mixed vigorously to release bacterial cells from the paper points. The paper points were removed and the suspension was centrifuged and re-suspended in 150 $\mu \mathrm{l}$ of TE. The samples were lysed by adding $150 \mu \mathrm{l}$ of $0.5 \mathrm{M} \mathrm{NaOH}$ and boiling for 10 minutes. Denatured DNA was neutralized with $800 \mu \mathrm{l}$ of $5 \mathrm{M} \mathrm{C}_{2} \mathrm{H}_{3} \mathrm{O}_{2} \mathrm{NH}_{4}$ and fixed in individual lanes on a nylon membrane (Hybond-N+, GE Healthcare Life Sciences, Piscataway, USA) using Minislot 30 (Immunetics, Cambridge, MA). The Miniblotter 45 apparatus (Immunetics) was used to hybridize 38 whole genomic DNA probes for 40 taxa of microorganisms. The probes were labeled with digoxigenin using the "Random Primer Digoxigenin Labeling Kit" (Roche Molecular Systems, Alameda, USA). DNA from serotypes $\mathrm{a}, \mathrm{b}$ and $\mathrm{c}$ of A. actinomycetemcomitans was pooled in one probe. Samples were analyzed by the checkerboard DNADNA hybridization technique, ${ }^{12}$ with modifications.

For the purpose of this study, the presence of six bacterial species at baseline was evaluated in prediction models. The three species comprising the red complex (Porphyromonas gingivalis, Tannerella forsythia and Treponema denticola), two species from the orange complex (Parvimonas micra and Prevotella intermedia) and Aggregatibacter actinomycetemcomitans were selected. Because of a highly skewed distribution of the data, microbial counts were analyzed as dichotomous variables. The presence of a specific species was determined using the cut-off point for detection of the checkerboard, established at $10^{4}$ cells. Failure to detect a sign was recorded as zero.

\section{Ethical aspects}

The study protocol was approved by the Committee of Ethical Affairs of the School of Dentistry of the UFRGS. The subjects read and signed an informed consent before entering the study.

\section{Statistical analysis}

A total of 257 teeth presenting both CA loss $\geq 4 \mathrm{~mm}$ and PPD $\geq 4 \mathrm{~mm}$ at the baseline examination were included in the analyses. The mean number of teeth presenting these clinical characteristics by patient was 10.7 (standard deviation: 6.6; median=10.5 teeth). The worst affected site of each tooth was used in the analysis.

The following variables were assessed as possible predictors: age (years), gender (male/female), smoking status (nonsmoker/smoker), use of systemic antibiotic (placebo/azithromycin), tooth type (molar/non-molar), disease extent (localized/generalized), time elapsed from baseline (time points), visible plaque at each time point (absent/present: presence of visible plaque on at least one site per tooth), and baseline PPD category (4-6 mm/ $\geq 7 \mathrm{~mm})$. The subjects were categorized into localized or generalized AgP as follows: ${ }^{13}$ subjects with at least one incisor or first molar with clinical attachment loss $\geq 4 \mathrm{~mm}$ and no more than two teeth other than the first molars or incisors were classified as having localized $\mathrm{AgP}$, whereas those with three or more affected non-incisors/first molars were classified as having generalized AgP. Separate prediction models were fitted for each bacterial species and for an additional variable generated to describe the number of concomitant species from the red complex detected in each individual (1, 2 or 3 species). These microbiological models were adjusted for other predictors.

The outcomes of the present study included mean changes in PPD, CA level and BOP over 12 months. Crude comparisons of changes over time were made using commands that take into account clustering of teeth within individuals, with a robust variance estimator (Huber/White/sandwich estimator of variance). Wald-test adjusted p-values were used for multiple comparisons over time.

Multivariable analysis using generalized estimating equations (GEE) with exchangeable correlation structure and a robust variance 
estimator was applied to evaluate the longitudinal effect of each predictor on PPD, CA loss and BOP, separately. GEE with Gaussian distribution and identity link was used. Univariable models were fitted, and predictors showing associations with $p$ values $\leq 0.25$ were entered in a multivariable model. Those predictors with non-significant associations $(p \geq 0.05)$ and demonstrating no confounding modification (at least $25 \%$ change in the other predictor estimates) were excluded from the final multivariable model. Interaction effects and multicollinearity were examined, and none were found to be statistically significant.

One subject from the placebo group moved out of town and missed the 12-month examination; a carry-forward strategy was employed for this individual, in order to account for the missing information, and the 9-month data were used following an intention-to-treat approach. Data analysis was performed using Stata software (Stata 14, Stata Corporation, College Station, USA).

\section{Results}

Twenty-eight individuals were screened for the study; however, 12 and 13 patients were randomized to the azithromycin and the placebo groups, respectively. One patient dropped out the study at the 3 month evaluation period for no specific reason, and was not included in the analysis (Figure 1). The sample characteristics at baseline are shown in Table 1 . The mean

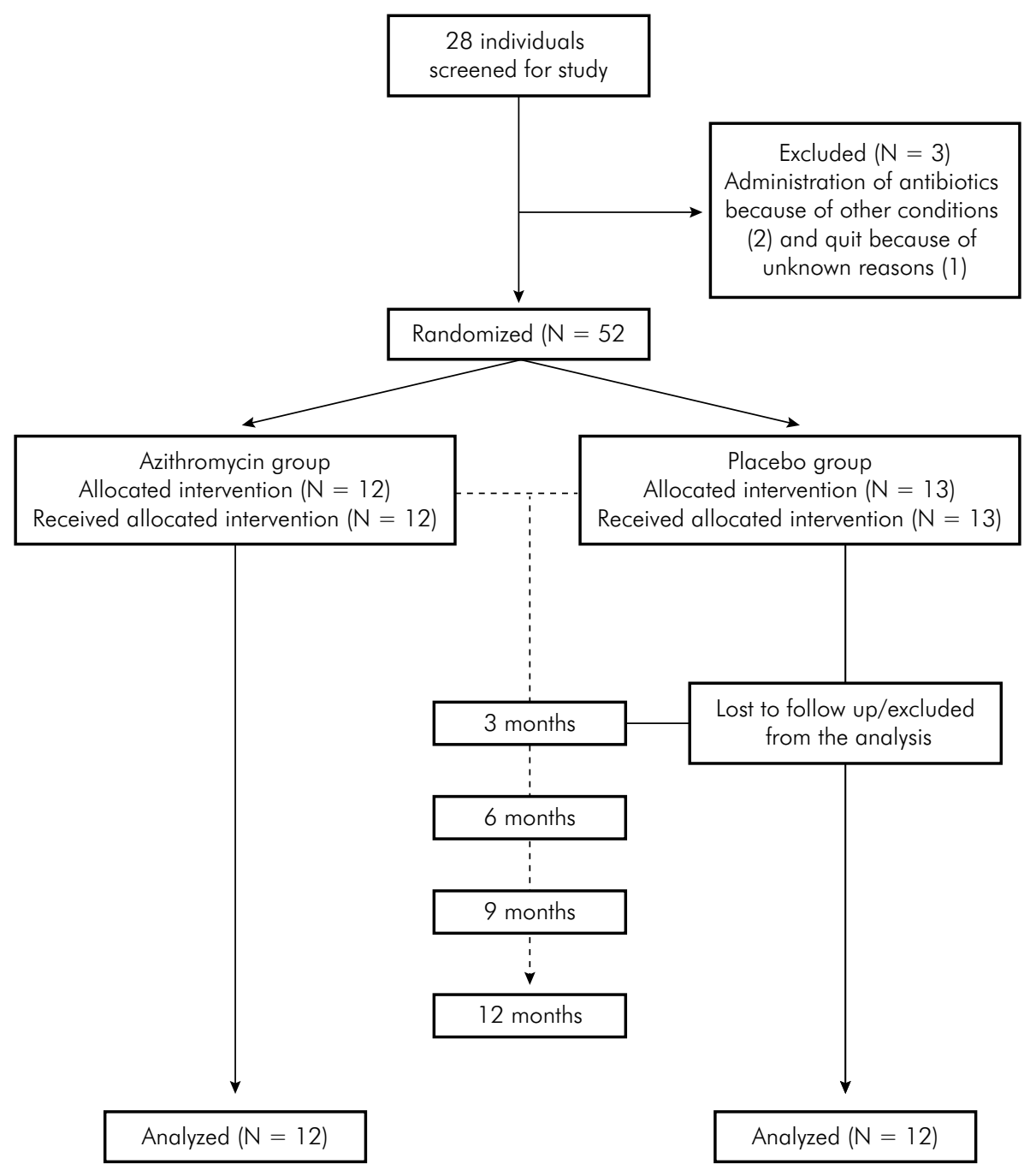

Figure 1. Flowchart of participation in the study 
Table 1. Study sample characteristics at baseline

\begin{tabular}{lccc}
\hline Variable & & Statistics & Estimate \\
\hline Age & Years & mean \pm SD & $21.3 \pm 3.7$ \\
Gender & Males & $\mathrm{n}(\%)$ & $13(54.2)$ \\
& Females & & $11(45.8)$ \\
Disease extent & Localized & $\mathrm{n}(\%)$ & $11(45.8)$ \\
& Generalized & & $13(54.2)$ \\
Smoking status & Nonsmokers & $\mathrm{n}(\%)$ & $19(79.2)$ \\
& Smokers & & $5(20.8)$ \\
Tooth type & Molars & $\mathrm{n}(\%)$ & $88(34.2)$ \\
& Non-molars & & $169(65.8)$ \\
Visible plaque & \% sites & mean $\pm S D$ & $71.5 \pm 29.8$ \\
PPD & & & \\
$4-6 \mathrm{~mm}$ & \% teeth & $\mathrm{n}(\%)$ & $127(49.4)$ \\
$\geq 7 \mathrm{~mm}$ & $\%$ teeth & $\mathrm{n}(\%)$ & $130(50.6)$ \\
CAL & & & \\
$4-6 \mathrm{~mm}$ & $\%$ teeth & $\mathrm{n}(\%)$ & $180(70.0)$ \\
$\geq 7 \mathrm{~mm}$ & \% teeth & $\mathrm{n}(\%)$ & $77(30.0)$ \\
BOP & \% sites & mean $\pm S D$ & $70.9 \pm 23.8$ \\
\hline
\end{tabular}

PPD: periodontal probing depth; CAL: clinical attachment level; BOP: bleeding on probing; SD: standard deviation

initial PPD and CA loss were $6.5 \mathrm{~mm}$ (SE: 0.3) and $5.8 \mathrm{~mm}$ (SE: 0.2), respectively. There was a significant reduction of $2.3 \mathrm{~mm}$ (SE: 0.2) in PPD and 48\% in the extent of sites with BOP, from baseline to three months after treatment. The mean CA gain was $1.2 \mathrm{~mm}$ (SE: 0.2) after the three first months. These improvements endured throughout the 12-month period.

Figure 2 shows the pattern of unadjusted mean PPD over the 12-month follow-up period, according to disease extent, use of antibiotics, tooth type and baseline PPD. In the multivariable model, these predictors showed significant effects on PPD change (Table 2). The reduction in PPD during 12 months was $0.58 \mathrm{~mm}$ higher in subjects with generalized versus localized AgP, $0.49 \mathrm{~mm}$ higher in subjects who had received adjunctive systemic azithromycin versus placebo, and $0.49 \mathrm{~mm}$ higher in non-molars versus molars. On the other hand, teeth with a baseline PPD $\geq 7 \mathrm{~mm}$ had a $1.32 \mathrm{~mm}$ higher PPD over time than teeth with a baseline PPD of 4-6 $\mathrm{mm}$. Most of the PPD reduction occurred during the first 3 months post-treatment, and there were minimal changes in PPD at 6, 9 and 12 months follow-up.

Unadjusted mean CA level according to visible plaque and baseline PPD is shown in Figure 3. Changes in CA level were significantly associated with these two predictors (Table 3). Teeth with no visible plaque during a given follow-up examination had a $0.22 \mathrm{~mm}$ higher CA gain, on average, than teeth with dental plaque. Teeth with PPD $\geq 7 \mathrm{~mm}$ at baseline had a $1.36 \mathrm{~mm}$ higher CA loss over 12 months than teeth with PPD 4-6 mm at baseline. The greatest amount of CA gain occurred in the first 3 months after treatment.

Presence of BOP was significantly associated with the use of azithromycin, plaque, baseline PPD and follow-up duration (Table 4). Use of azithromycin predicted a $14 \%$ reduction in the percentage of sites with BOP. Local factors such as tooth type and visible plaque accounted for small but significant contributions. Deep pockets at baseline predicted a $7 \%$ higher BOP than sites with baseline pockets of 4-6 mm.

None of the six bacterial species assessed in this study significantly predicted the changes in CA level, PPD and BOP during 12 months (Table 5). The presence of all three species of the red complex predicted a $0.78 \mathrm{~mm}$ higher CA loss over the 12 months, compared with the presence of only one species $(p=0.02)$.

\section{Discussion}

The present study explored patient- and tooth-related factors, such as predictors of the clinical response to nonsurgical periodontal therapy among young individuals with AgP. Overall, the factors that consistently predicted clinical outcomes included disease extent, adjunctive use of azithromycin, tooth type, presence of visible plaque, and baseline level of PPD. Most of these variables showed statistically significant prediction of the reduction in PPD and $\mathrm{BOP}$, whereas fewer variables showed significant predictions of CA gain.

Limited data exist on the wound healing of subjects with AgP; however, it appears from the literature that generalized AgP is more challenging to treat than localized AgP. ${ }^{3}$ Notably, in this study, subjects with generalized AgP showed greater PPD reduction than those with the localized form of the disease. We found no indication that this disparity may be attributed to differences in PPD at baseline between the groups (generalized: $6.59 \pm 0.26 \mathrm{~mm} v s$. localized: $6.20 \pm 0.55 \mathrm{~mm}, \mathrm{p}=0.53$ ). Reasons for this finding are still unknown and can only be speculated. 

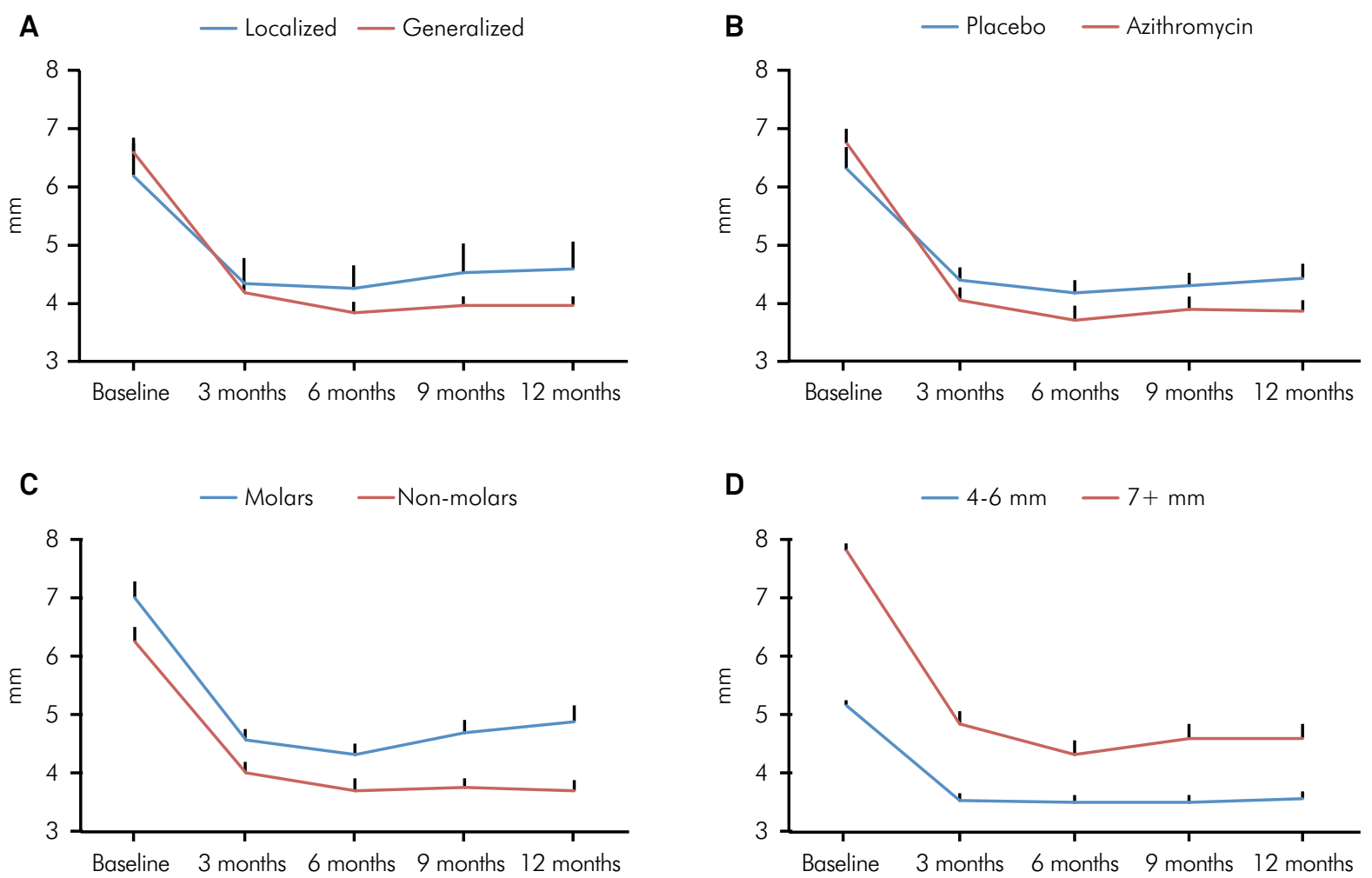

Figure 2. Periodontal probing depth over 12 months, according to different predictors (a: disease extent; b: antibiotic use; c: tooth type; $d$ : baseline PPD)

Table 2. Univariable and multivariable prediction models of PPD change over 12 months

\begin{tabular}{|c|c|c|c|c|c|c|c|}
\hline \multirow{2}{*}{ Predictor } & & \multicolumn{3}{|c|}{ Univariable } & \multicolumn{3}{|c|}{ Multivariable } \\
\hline & & Coef. & SE & $p$ & Coef. & SE & $p$ \\
\hline Patient-related factors & & - & - & - & - & - & - \\
\hline Age & Years & -0.02 & 0.05 & 0.73 & - & - & - \\
\hline \multirow{2}{*}{ Gender } & Males & Ref. & - & - & - & - & - \\
\hline & Females & -0.09 & 0.38 & 0.80 & - & - & - \\
\hline \multirow{2}{*}{ Disease extent } & Localized & Ref. & - & - & Ref. & - & - \\
\hline & Generalized & -0.42 & 0.45 & 0.12 & -0.58 & 0.28 & 0.04 \\
\hline \multirow{2}{*}{ Smoking status } & Nonsmokers & Ref. & - & - & - & - & - \\
\hline & Smokers & 0.002 & 0.33 & 0.99 & - & - & - \\
\hline \multirow{2}{*}{ Antibiotic use } & Placebo & Ref. & - & - & Ref. & - & - \\
\hline & Azithromycin & -0.43 & 0.38 & 0.24 & -0.49 & 0.24 & 0.04 \\
\hline \multicolumn{8}{|l|}{ Tooth-related factors } \\
\hline \multirow{2}{*}{ Tooth type } & Molars & Ref. & - & - & Ref. & - & - \\
\hline & Non-molars & -0.69 & 0.20 & 0.001 & -0.49 & 0.16 & 0.002 \\
\hline \multirow{2}{*}{ Visible plaque } & Present & Ref. & - & - & - & - & - \\
\hline & Absent & -0.80 & 0.17 & $<0.001$ & - & - & - \\
\hline \multirow{2}{*}{ Baseline PPD } & $4-6 \mathrm{~mm}$ & Ref. & - & - & Ref. & - & - \\
\hline & $\geq 7 \mathrm{~mm}$ & 1.39 & 0.14 & $<0.001$ & 1.32 & 0.12 & $<0.001$ \\
\hline \multirow{5}{*}{ Time } & Baseline & Ref. & - & - & Ref. & & - \\
\hline & 3 months & -2.32 & 0.22 & $<0.001$ & -2.31 & 0.22 & $<0.001$ \\
\hline & 6 months & -2.60 & 0.29 & $<0.001$ & -2.59 & 0.29 & $<0.001$ \\
\hline & 9 months & -2.45 & 0.24 & $<0.001$ & -2.45 & 0.24 & $<0.001$ \\
\hline & 12 months & -2.41 & 0.25 & $<0.001$ & -2.42 & 0.25 & $<0.001$ \\
\hline
\end{tabular}

PPD: periodontal probing depth; Coef.: coefficient; SE: standard error; Ref.: reference category 
Table 3. Univariable and multivariable prediction models of CA level change over 12 months.

\begin{tabular}{|c|c|c|c|c|c|c|c|}
\hline \multirow{2}{*}{ Predictor } & & \multicolumn{3}{|c|}{ Univariable } & \multicolumn{3}{|c|}{ Multivariable } \\
\hline & & Coef. & SE & $p$ & Coef. & SE & $p$ \\
\hline \multicolumn{8}{|c|}{ Patient-related factors } \\
\hline Age & Years & 0.03 & 0.09 & 0.77 & - & - & - \\
\hline \multirow{2}{*}{ Gender } & Males & Ref. & - & - & - & - & - \\
\hline & Females & 0.13 & 0.47 & 0.79 & - & - & - \\
\hline \multirow{2}{*}{ Disease extent } & Localized & Ref. & - & - & - & - & - \\
\hline & Generalized & 0.08 & 0.50 & 0.87 & - & - & - \\
\hline \multirow{2}{*}{ Smoking status } & Nonsmokers & Ref. & & & - & - & - \\
\hline & Smokers & 0.31 & 0.38 & 0.42 & - & - & - \\
\hline \multirow{2}{*}{ Antibiotic use } & Placebo & Ref. & - & - & - & - & - \\
\hline & Azithromycin & -0.11 & 0.47 & 0.82 & - & - & - \\
\hline \multicolumn{8}{|c|}{ Tooth-related factors } \\
\hline Tooth type & $\begin{array}{c}\text { Molars } \\
\text { Non-molars }\end{array}$ & $\begin{array}{l}\text { Ref. } \\
0.01\end{array}$ & 0.32 & 0.97 & - & - & - \\
\hline \multirow{2}{*}{ Visible plaque } & Present & Ref. & - & - & Ref & - & - \\
\hline & Absent & -0.70 & 0.13 & $<0.001$ & -0.22 & 0.11 & 0.04 \\
\hline \multirow{2}{*}{ Baseline PPD } & $4-6 \mathrm{~mm}$ & Ref. & - & - & Ref & - & - \\
\hline & $\geq 7 \mathrm{~mm}$ & 1.38 & 0.20 & $<0.001$ & 1.36 & 0.20 & $<0.001$ \\
\hline \multirow{5}{*}{ Time } & Baseline & Ref & - & - & Ref. & - & - \\
\hline & 3 months & -1.25 & 0.18 & $<0.001$ & -1.16 & 0.19 & $<0.001$ \\
\hline & 6 months & -1.56 & 0.19 & $<0.001$ & -1.49 & 0.19 & $<0.001$ \\
\hline & 9 months & -1.43 & 0.20 & $<0.001$ & -1.37 & 0.20 & $<0.001$ \\
\hline & 12 months & -1.40 & 0.18 & $<0.001$ & -1.33 & 0.19 & $<0.001$ \\
\hline
\end{tabular}

PPD: periodontal probing depth; Coef.: coefficient; SE: standard error; Ref.: reference category

A

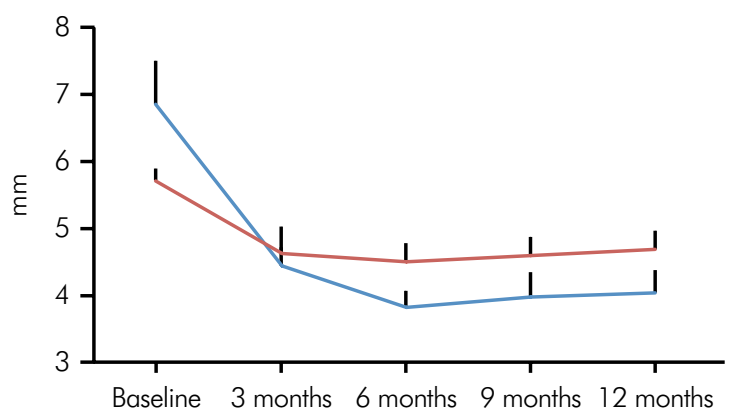

B $\quad-4-6 \mathrm{~mm} \quad-7+\mathrm{mm}$

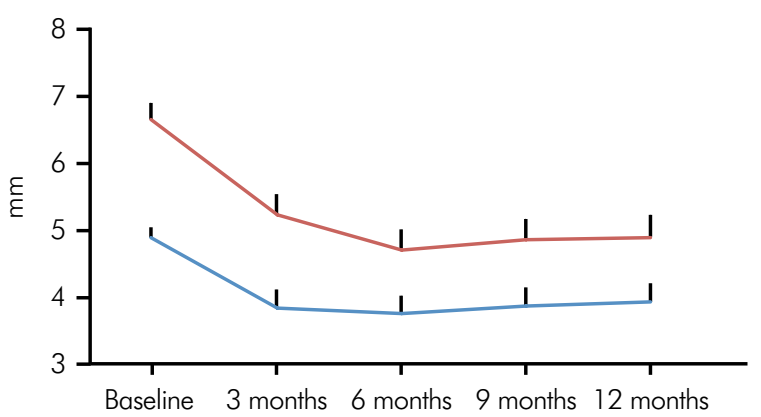

Figure 3. Clinical attachment level over 12 months, according to different predictors (a: visible plaque; b: baseline PPD).

Smoking is considered a major risk factor of periodontitis. ${ }^{14}$ Smoking is also a well-known predictor of poor response to periodontal therapy, ${ }^{15}$ but few studies have further demonstrated that smoking negatively impacts treatment outcomes in subjects with $\mathrm{AgP}{ }^{6,9}$ In the present study, smoking did not show a significant effect on the non-surgical treatment outcome of AgP. However, only 5 of the subjects (or $21 \%$ ) were smokers, and this may have mitigated the effect of this variable in this study.
Although it is well-established that good plaque control is important for periodontal treatment success, ${ }^{16}$ scant data exist regarding its impact on AgP treatment. Recent studies suggest that local factors and poor oral hygiene are associated with AgP. ${ }^{2}$ The findings of the present study support the importance of adequate oral hygiene in the management of AgP. Absence of plaque at tooth sites was a significant predictor of higher CA gain and $\mathrm{BOP}$ reduction. 
Predictors of clinical outcomes after periodontal treatment of aggressive periodontitis: 12-month randomized trial

Table 4. Univariable and multivariable prediction models of BOP change over 12 months.

\begin{tabular}{|c|c|c|c|c|c|c|c|}
\hline \multirow{2}{*}{ Predictor } & \multirow{2}{*}{-} & \multicolumn{3}{|c|}{ Univariable } & \multicolumn{3}{|c|}{ Multivariable } \\
\hline & & Coef. & SE & $p$ & Coef. & SE & $p$ \\
\hline \multicolumn{8}{|c|}{ Patient-related factors } \\
\hline Age & Years & -0.001 & 0.01 & 0.82 & - & - & - \\
\hline \multirow[t]{2}{*}{ Gender } & Male & Ref. & - & - & - & - & - \\
\hline & Female & 0.009 & 0.05 & 0.86 & - & - & - \\
\hline \multirow[t]{2}{*}{ Disease extent } & Localized & Ref. & - & - & - & - & - \\
\hline & Generalized & -0.04 & 0.05 & 0.43 & - & - & - \\
\hline \multirow[t]{2}{*}{ Smoking status } & Nonsmokers & Ref. & - & - & - & - & - \\
\hline & Smokers & -0.02 & 0.04 & 0.57 & - & - & - \\
\hline \multirow[t]{2}{*}{ Antibiotic use } & Placebo & Ref. & - & - & Ref. & - & - \\
\hline & Azithromycin & -0.13 & 0.04 & 0.002 & -0.14 & 0.04 & 0.001 \\
\hline \multicolumn{8}{|c|}{ Tooth-related factors } \\
\hline \multirow[t]{2}{*}{ Tooth type } & Molars & Ref. & - & - & Ref. & & \\
\hline & Non-molars & -0.06 & 0.02 & 0.001 & -0.05 & 0.02 & 0.01 \\
\hline \multirow[t]{2}{*}{ Visible plaque } & Present & Ref. & - & - & Ref. & - & - \\
\hline & Absent & -0.21 & 0.02 & $<0.001$ & -0.06 & 0.02 & 0.002 \\
\hline \multirow[t]{4}{*}{ Baseline PPD } & $4-6 \mathrm{~mm}$ & Ref. & - & - & Ref. & - & - \\
\hline & $\geq 7 \mathrm{~mm}$ & 0.08 & 0.02 & $<0.001$ & 0.07 & 0.02 & $<0.001$ \\
\hline & Baseline & Ref. & - & - & Ref. & - & - \\
\hline & 3 months & -0.48 & 0.03 & $<0.001$ & -0.46 & 0.03 & $<0.001$ \\
\hline \multirow[t]{3}{*}{ Time } & 6 months & -0.53 & 0.03 & $<0.001$ & -0.51 & 0.03 & $<0.001$ \\
\hline & 9 months & -0.47 & 0.03 & $<0.001$ & -0.45 & 0.03 & $<0.001$ \\
\hline & 12 months & -0.45 & 0.03 & $<0.001$ & -0.43 & 0.03 & $<0.001$ \\
\hline
\end{tabular}

PPD: periodontal probing depth; Coef.: coefficient; SE: standard error; Ref.: reference category

Table 5. Multivariable GEE models" of the presence of various bacterial species at baseline as predictors of CA loss, PPD and BOP changes over 12 months.

\begin{tabular}{|c|c|c|c|c|c|c|c|c|c|}
\hline \multirow{2}{*}{ Predictor } & \multicolumn{3}{|c|}{ CA loss* } & \multicolumn{3}{|l|}{$\mathrm{PPD}^{* *}$} & \multicolumn{3}{|l|}{$\mathrm{BOP} * * *$} \\
\hline & Coef. & SE & $\mathrm{p}$ & Coef. & SE & $p$ & Coef. & SE & $\mathrm{p}$ \\
\hline P. gingivalis & 0.40 & 0.40 & 0.32 & 0.17 & 0.24 & 0.70 & 0.02 & 0.04 & 0.55 \\
\hline T. forsythia & 0.47 & 0.36 & 0.19 & 0.01 & 0.22 & 0.98 & -0.002 & 0.04 & 0.96 \\
\hline T. denticola & 0.22 & 0.56 & 0.70 & -0.12 & 0.38 & 0.75 & 0.04 & 0.05 & 0.42 \\
\hline A. actinomycetemcomitans & -0.38 & 0.43 & 0.37 & -0.43 & 0.32 & 0.18 & -0.04 & 0.05 & 0.46 \\
\hline P. micra & 0.35 & 0.38 & 0.36 & -0.13 & 0.23 & 0.58 & 0.02 & 0.04 & 0.96 \\
\hline P. intermedia & 0.29 & 0.40 & 0.47 & -0.16 & 0.28 & 0.58 & 0.01 & 0.04 & 0.75 \\
\hline \multicolumn{10}{|l|}{ Red complex } \\
\hline 1 species & Ref. & & & Ref. & & & Ref. & & \\
\hline 2 species & 0.10 & 0.53 & 0.84 & -0.11 & 0.32 & 0.74 & -0.05 & 0.04 & 0.26 \\
\hline 3 species & 0.78 & 0.33 & 0.02 & 0.17 & 0.33 & 0.60 & 0.02 & 0.05 & 0.63 \\
\hline
\end{tabular}

\#Each bacterial species was evaluated in separate models for CA loss, PPD and BOP

*Adjusted for plaque, time and baseline PPD

** Adjusted for time, antibiotic use, tooth type, disease extent and baseline PPD

*** Adjusted for time, antibiotic use, plaque, tooth type and baseline PPD 
The benefits of the adjunctive use of antibiotics in the treatment of destructive periodontal diseases have been demonstrated. ${ }^{17,18}$ However, the efficacy of classic chemotherapeutic agents has been influenced by their complex dosing schedule, low compliance, development of bacterial resistance, and other side effects. ${ }^{17}$ In contrast, azithromycin has a convenient dosing schedule, thus potentially increasing compliance, ${ }^{19}$ and is effective against major periodontopathogens. ${ }^{20,21}$ The efficacy of adjunctive azithromycin in the nonsurgical treatment of $\mathrm{AgP}$ was first reported in 2008. ${ }^{11}$ In the present study, azithromycin showed more favorable treatment outcomes for PPD and BOP in multivariable prediction models.

Periodontal treatment of molars is more complex than that of single-rooted teeth. In AgP patients, molars are the most frequently lost teeth. ${ }^{8}$ In this study, non-molars had a higher PPD and BOP reduction than molars, whereas CA level changes were not different by tooth type. These findings are in agreement with Baumer et al., ${ }^{6}$ who reported higher tooth loss rates in molars after periodontal therapy. In contrast, Hughes et al. ${ }^{9}$ did not find any significant differences in treatment response between single- and multi-rooted teeth.

In this analysis, deep pockets at baseline were associated with a smaller decrease in PPD and a higher CA loss than sites with moderate PPD. This finding could seem counterintuitive, since it has been demonstrated that deep pockets have a higher potential for improvement..$^{10}$ The reason could be that final mean PPD and CA loss was still higher for teeth with initially deep versus moderate pockets (3.6 $\mathrm{mm}$ and $4.6 \mathrm{~mm}$, respectively). Thus, although greater improvement is likely to be achieved in teeth with deeper pockets, these teeth will still present the worst post-treatment parameters.

The presence of high proportions of putative periodontal pathogens, such as the red complex species (P. gingivalis, T. forsythia and T. denticola) and $A$. actinomycetemcomitans, has been associated with the progression of clinical attachment loss; therefore, failure to reduce specific pathogens may lead to disease recurrence. ${ }^{7,22,23}$ In the current investigation, the presence of all three members of the red complex at baseline, adjusted for other factors, was associated with a higher CA loss over the 12 months after treatment. In interpreting these data, one should be careful to take into consideration the microbiological assessment applied, namely a pooled subgingival sample of each individual's biofilm, which assessed the presence/absence of bacteria at a specific threshold for each sample pooled. Nonetheless, the present findings indicate that identification of the microbiological profile of AgP patients before treatment has a possible clinical application.

A possible shortcoming of this study was the relatively small number of individuals. Consequently, some variables could have been overlooked, because of the restrictions in the sample size. In other words, some predictors may not have demonstrated significant associations that could have been found if the sample were larger, one example being smoking; clinicians should keep this in mind when interpreting the findings. Nevertheless, it is expected that future studies with AgP patients will present with fewer individuals, due to the lower AgP prevalence in the population, compared with other forms of periodontitis. ${ }^{24}$ Studies evaluating different types of periodontal interventions in AgP patients have also used sample sizes similar to those of the present randomized trial..$^{25,26,27}$

Future prospects for research in this field include studies with longer follow-up periods and larger sample sizes. The new information provided in this study also highlights the importance of multivariable analyses in clinical trial testing interventions for $\mathrm{AgP}$, in order to account for a possible confounding bias of the predictors detected herein. From a clinical perspective, the present data provide information for the clinical management of AgP, aiming to achieve better clinical outcomes following non-surgical treatment.

\section{Conclusion}

Patient- and tooth-related factors predicting better treatment outcomes of $\mathrm{AgP}$ included extent of periodontitis, use of azithromycin, type of teeth affected, plaque, and baseline PPD. The prediction of CA level changes was more challenging, and the absence of plaque and baseline PPD were the only significant predictors of CA gain. The concomitant presence of periodontal pathogens of the red complex in the subgingival biofilm may indicate challenges in ultimately achieving a favorable treatment outcome for AgP. 


\section{References}

1. Corraini P, Pannuti CM, Pustiglioni AN, Romito GA, Pustiglioni FE. Risk indicators for aggressive periodontitis in an untreated isolated young population from Brazil. Braz Oral Res. 2009;23(2):209-15. doi:10.1590/S1806-83242009000200019

2. Susin C, Albandar JM. Aggressive periodontitis in an urban population in southern Brazil. J Periodontol. 2005;76(3):468-75. doi:10.1902/jop.2005.76.3.468

3. Deas DE, Mealey BL. Response of chronic and aggressive periodontitis to treatment. Periodontol 2000. 2010;53(1):154-66. doi:10.1111/j.1600-0757.2009.00334.x

4. Taiete T, Casati MZ, Ribeiro EP, Sallum EA, Nociti Júnior FH, Casarin RCV. Amoxicillin/metronidazole associated with nonsurgical therapy did not promote additional benefits in immunologic parameters in generalized aggressive periodontitis: a randomized controlled clinical trial. Quintessence Int. 2015 Sep 4. doi:10.3290/j.qi.a34723

5. Rabelo CC, Feres M, Gonçalves C, Figueiredo LC, Faveri M, Tu $\mathrm{YK}$, et al. Systemic antibiotics in the treatment of aggressive periodontitis. A systematic review and a Bayesian Network metaanalysis. J Clin Periodontol. 2015;42(7):647-57. doi:10.1111/jcpe.12427

6. Bäumer A, El Sayed N, Kim TS, Reitmeir P, Eickholz P, Pretzl B. Patient-related risk factors for tooth loss in aggressive periodontitis after active periodontal therapy. J Clin Periodontol. 2011;38(4):347-54. doi:10.1111/j.1600-051X.2011.01698.x

7. Kamma JJ, Baehni PC. Five-year maintenance follow-up of early-onset periodontitis patients. J Clin Periodontol. 2003;30(6):562-72. doi:10.1034/j.1600-051X.2003.00289.x

8. Bäumer A, Pretzl B, Cosgarea R, Kim TS, Reitmeir P, Eickholz $\mathrm{P}$, et al. Tooth loss in aggressive periodontitis after active periodontal therapy: patient-related and tooth-related prognostic factors. J Clin Periodontol. 2011;38(7):644-51. doi:10.1111/j.1600-051X.2011.01733.x

9. Hughes FJ, Syed M, Koshy B, Marinho V, Bostanci N, McKay IJ, et al. Prognostic factors in the treatment of generalized aggressive periodontitis: I. Clinical features and initial outcome. J Clin Periodontol. 2006;33(9):663-70. doi:10.1111/j.1600-051X.2006.00966.x

10. Heitz-Mayfield LJA, Trombelli L, Heitz F, Needleman I, Moles D. A systematic review of the effect of surgical debridement vs non-surgical debridement for the treatment of chronic periodontitis. J Clin Periodontol. 2002;29(Suppl s3):92-102. doi:10.1034/j.1600-051X.29.s3.5.x

11. Haas AN, Castro GD, Moreno T, Susin C, Albandar JM, Oppermann RV, et al. Azithromycin as an adjunctive treatment of aggressive periodontitis: 12-months randomized clinical trial. J Clin Periodontol. 2008;35(8):696-704. doi:10.1111/j.1600-051X.2008.01254.x

12. Socransky SS, Smith C, Martin L, Paster BJ, Dewhirst FE, Levin AE. Checkerboard DNA-DNA hybridization. Biotechniques. 1994;17(4):788-92.
13. Armitage GC. Development of a classification system for periodontal diseases and conditions. Ann Periodontol. 1999;4(1):1-6. doi:10.1902/annals.1999.4.1.1

14. Bergström J. Tobacco smoking and risk for periodontal disease. J Clin Periodontol. 2003;30(2):107-13. doi:10.1034/j.1600-051X.2003.00272.x

15. Wan CP, Leung WK, Wong MC, Wong RM, Wan $\mathrm{P}$, Lo EC, et al. Effects of smoking on healing response to non-surgical periodontal therapy: a multilevel modelling analysis. J Clin Periodontol. 2009;36(3):229-39. doi:10.1111/j.1600-051X.2008.01371.x

16. Rosling B, Nyman S, Lindhe J, Jern B. The healing potential of the periodontal tissues following different techniques of periodontal surgery in plaque-free dentitions. A 2-year clinical study. J Clin Periodontol. 1976;3(4):233-50. doi:10.1111/j.1600-051X.1976.tb00042.x

17. Sgolastra F, Petrucci A, Gatto R, Monaco A. Effectiveness of systemic amoxicillin/metronidazole as an adjunctive therapy to full-mouth scaling and root planing in the treatment of aggressive periodontitis: a systematic review and meta-analysis. J Periodontol. 2012;83(6):731-43. doi:10.1902/jop.2011.110432

18. Herrera D, Alonso B, León R, RoldánS, Sanz M. Antimicrobial therapy in periodontitis: the use of systemic antimicrobials against the subgingival biofilm. J Clin Periodontol. 2008;35(8 Suppl):45-66. doi:10.1111/j.1600-051X.2008.01260.x

19. Foulds G, Shepard RM, Johnson RB. The pharmacokinetics of azithromycin in human serum and tissues. J Antimicrob Chemother. 1990;25(Suppl A):73-82. doi:10.1093/jac/25.suppl_A.73

20. Pajukanta R, Asikainen S, Saarela M, Alaluusua S, Jousimies-Somer H. In vitro activity of azithromycin compared with that of erythromycin against Actinobacillus actinomycetemcomitans. Antimicrob Agents Chemother. 1992;36(6):1241-3. doi:10.1128/AAC.36.6.1241

21. Pajukanta R. In vitro antimicrobial susceptibility of Porphyromonas gingivalis to azithromycin, a novel macrolide. Oral Microbiol Immunol. 1993;8(5):325-6. doi:10.1111/j.1399-302X.1993.tb00583.x

22. Heller D, Varela VM, Silva-Senem MX, Torres MC, Feres-Filho EJ, Colombo AP. Impact of systemic antimicrobials combined with anti-infective mechanical debridement on the microbiota of generalized aggressive periodontitis: a 6-month RCT. J Clin Periodontol. 2011;38(4):355-64. doi:10.1111/j.1600-051X.2011.01707.x

23. Silva-Senem MX, Heller D, Varela VM, Torres MC, Feres-Filho EJ, Colombo AP. Clinical and microbiological effects of systemic antimicrobials combined to an antiinfective mechanical debridement for the management of aggressive periodontitis: a 12-month randomized controlled trial. J Clin Periodontol. 2013;40(3):242-51. doi:10.1111/ jcpe.12052 
24. Susin C, Haas AN, Albandar JM. Epidemiology and demographics of aggressive periodontitis. Periodontol 2000. 2014;65(1):27-45. doi:10.1111/prd.12019

25. Saxén L, Asikainen S. Metronidazole in the treatment of localized juvenile periodontitis. J Clin Periodontol. 1993;20(3):166-71. doi:10.1111/j.1600-051X.1993.tb00339.x

26. Tinoco EM, Beldi MI, Campedelli F, Lana M, Loureiro CA, Bellini HT, et al. Clinical and microbiological effects of adjunctive antibiotics in treatment of localized juvenile periodontitis. A controlled clinical trial. J Periodontol. 1998;69(12):1355-63. doi:10.1902/jop.1998.69.12.1355

27. Mestnik MJ, Feres M, Figueiredo LC, Soares G, Teles RP, Fermiano $\mathrm{D}$, et al. The effects of adjunctive metronidazole plus amoxicillin in the treatment of generalized aggressive periodontitis: a 1-year double-blinded, placebo-controlled, randomized clinical trial.JClin Periodontol. 2012;39(10):955-61. doi:10.1111/j.1600-051X.2012.01932.x 